\title{
Article \\ On the Increasing Convex Order of Relative Spacings of Order Statistics
}

\author{
Antonia Castaño-Martínez, Gema Pigueiras * (D) and Miguel A. Sordo $\mathbb{D}$ \\ Dpto. Estadística e Investigación Operativa, Universidad de Cádiz, 11510 Puerto Real, Spain; \\ antonia.castano@uca.es (A.C.-M.); mangel.sordo@uca.es (M.A.S.) \\ * Correspondence: gema.pigueiras@uca.es
}

check for updates

Citation: Castaño-Martínez, A.; Pigueiras, G.; Sordo, M.A. On the Increasing Convex Order of Relative Spacings of Order Statistics. Mathematics 2021, 9, 618. https:// doi.org/10.3390/math9060618

Academic Editors: Antonio Di Crescenzo and Lev Klebanov

Received: 19 February 2021

Accepted: 11 March 2021

Published: 15 March 2021

Publisher's Note: MDPI stays neutral with regard to jurisdictional claims in published maps and institutional affiliations.

Copyright: (c) 2021 by the authors. Licensee MDPI, Basel, Switzerland. This article is an open access article distributed under the terms and conditions of the Creative Commons Attribution (CC BY) license (https:// creativecommons.org/licenses/by/ $4.0 /)$.

\begin{abstract}
Relative spacings are relative differences between order statistics. In this context, we extend previous results concerning the increasing convex order of relative spacings of two distributions from the case of consecutive spacings to general spacings. The sufficient conditions are given in terms of the expected proportional shortfall order. As an application, we compare relative deprivation within some parametric families of income distributions.
\end{abstract}

Keywords: order statistics; expected proportional shortfall order; relative spacings; deprivation

\section{Introduction}

Let $X_{1}, X_{2}, \ldots, X_{n}$ be $n$ independent and identically distributed (i.i.d.) random variables, and let $X_{1: n}, \ldots, X_{n: n}$ be the corresponding order statistics, which are the $X_{i}$ s arranged in a non-decreasing order (that is, $X_{1: n} \leq \ldots \leq X_{n: n}$ ). Differences between order statistics are called spacings. The sequences of spacings, given by

$$
S_{i, j, n}^{X}=X_{j: n}-X_{i: n}, \quad 1 \leq i<j \leq n,
$$

and relative spacings, given by

$$
R_{i, j, n}^{X}=\frac{X_{j: n}-X_{i: n}}{X_{i: n}}, \quad 1 \leq i<j \leq n,
$$

have found applicability in fields such as reliability, auction theory, insurance, economics and other areas of applied mathematics. Given two probability models, it is often of interest to study which one has larger spacings under different probabilistic criteria (see, e.g., Kochar [1], Kochar and Rojo [2], $\mathrm{Hu}$ and Wei [3], Kochar et al. [4], Kochar and $\mathrm{Xu}$ [5], Genest et al. [6], Mao and Hu [7], Zhao and Zhang [8], Torrado and Lillo [9], Belzunce et al. [10] and Ding et al. [11]). There has been less research conducted on the comparison of relative spacings, which is the focus of this paper. As far as we know, the earliest reference on this subject is the work of Belzunce et al. [12], who gave conditions for the comparison of relative spacings between consecutive generalized order statistics from two random vectors in terms of several stochastic orders. Some of these conditions were given in terms of the expected proportional shortfall order and the increasing convex order, which are defined below.

Definition 1. Let $X$ and $Y$ be two random variables with respective distribution functions $F$ and $G$ and survival functions $\bar{F}$ and $\bar{G}$, respectively. Let $F^{-1}$ and $G^{-1}$ be the corresponding quantile functions given by $F^{-1}(u)=\inf \{x: F(x) \geq u\}$ and $G^{-1}(u)=\inf \{x: G(x) \geq u\}$, for $0 \leq u \leq 1$. Then,

(a) We say that $X$ is less than $Y$ in the increasing convex order (denoted by $X \leq_{i c x} Y$ ) if

$$
E[\phi(X)] \leq E[\phi(Y)]
$$


for all increasing convex function $\phi: \mathbb{R} \longrightarrow \mathbb{R}$ or, equivalently, if

$$
\int_{s}^{+\infty} \bar{F}(t) d t \leq \int_{s}^{+\infty} \bar{G}(t) d t, \text { for all s. }
$$

(b) For non-negative random variables $X$ and $Y$ with finite means, we say that $X$ is less than $Y$ in the expected proportional shortfall order (denoted by $X \leq_{p s} Y$ ) if

$$
\frac{\int_{F^{-1}(p)}^{\infty} \bar{F}(t) d t}{F^{-1}(p)} \leq \frac{\int_{G^{-1}(p)}^{\infty} \bar{G}(t) d t}{G^{-1}(p)} \text { for all } p \in(0,1) .
$$

Many references and applications of the increasing convex order can be found in the books by Shaked and Shanthikumar [13] and Belzunce et al. [14]. References on the expected proportional shortfall include Belzunce et al. [12,15,16], Di Crescenzo et al. [17], Belzunce and Martínez-Riquelme [18], Sordo et al. [19], Arnold and Sarabia [20], and Arriaza et al. [21]. In particular, given two random variables $X$ and $Y$, Belzunce et al. [12] proved (considering spacings of generalized order statistics) that

$$
X_{1: n} \leq_{p s} Y_{1: n} \Longrightarrow \frac{X_{i+1: n}-X_{i: n}}{X_{i: n}} \leq_{i c x} \frac{Y_{i+1: n}-Y_{i: n}}{Y_{i: n}}, \quad 1 \leq i \leq n-1 .
$$

However, in many applied situations, relative $(i, j)$-spacings of the form (2) (and not only consecutive spacings where $j=i+1$ ) must be compared. One such situation is in the framework of deprivation theory. The notion of individual relative deprivation refers to the frustration felt by an individual in society when she/he compares herself/himself to more successful individuals (see Runciman [22]). If we consider a society with $n$ individuals who are identical except for their incomes, $x_{1: n} \leq \ldots \leq x_{n: n}$, the relative deprivation of an individual with rank $k$ when she/he compares herself/himself to a richer individual with rank $j$ can be quantified by the number $\left(\frac{x_{j: n}-x_{k: n}}{x_{k: n}}\right)$. The sum of these values across richer individuals (normalized by the population size) given by

$$
\frac{1}{n} \sum_{j=k+1}^{n}\left(\frac{x_{j: n}-x_{k: n}}{x_{k: n}}\right)
$$

represents their total relative deprivation (see, e.g., Paul [23], Chakravarty and Moyes [24] and Chakravarty et al. [25]). Given an income random variable $X$ with distribution function $F$, the theoretical version of (4) is given by

$$
R D P_{k, n}^{X}=\frac{1}{n} \sum_{j=k+1}^{n}\left(\frac{X_{j: n}-X_{k: n}}{X_{k: n}}\right)=\frac{1}{n} \sum_{j=k+1}^{n} R_{k, j, n}^{X},
$$

where $X_{1: n}, \ldots, X_{n: n}$ are the corresponding order statistics. Given another income random variable $Y$, a natural question is to find conditions under which

$$
E\left[R D P_{k, n}^{X}\right] \leq E\left[R D P_{k, n}^{Y}\right], \text { for all } 1 \leq k \leq n
$$

a condition that means there is no more relative deprivation under $X$ than under $Y$ (see Chakravarty and Moyes [24]) and that cannot be deduced from (3).

In this paper, we generalize (3) providing conditions under which

$$
R_{i, j, n}^{X} \leq_{i c x} R_{i, j, n}^{Y} \text { for } 1 \leq i<j \leq n
$$

Two points should be noted here. First, (5) does not follow from the results in Belzunce et al. [12] and new research has been conducted to achieve sufficient conditions for these orderings; second, the order $\leq_{i c x}$ is much more informative than just a simple 
comparison of expectations, since it allows one to compare increasing convex functions of relative spacings.

To this aim, the paper is organized as follows: In Section 2, we provide conditions to compare order statistics arising from two random variables in terms of the expected proportional shortfall order. The purpose is to obtain sufficient conditions to compare relative spacings (from two random variables) that are not necessarily adjacent in terms of the increasing convex order. We also provide a sufficient condition, in terms of spacings, for stochastic equality (up to a scale parameter) under the expected proportional shortfall ordering. In Section 3, we apply the results to the comparison of income distributions in terms of relative deprivation. The income distributions under study are the generalized gamma and generalized beta of the second kind, which include, as particular cases, some of the most popular distributions used in the analysis of income. In this section, the relationship between the expected proportional shortfall order and the star-shaped order, which is defined as follows, is exploited.

Definition 2. Let $X$ and $Y$ be two random variables with respective distribution functions $F$ and $G$. We say that $X$ is less than $Y$ in the star-shaped order (denoted by $X \leq_{*} Y$ ) if and only if

$$
\frac{G^{-1}(p)}{F^{-1}(p)} \text { is increasing in } p \in(0,1) \text {. }
$$

Recent applications of the star-shaped order can be found in Arriaza et al. [21] and the book by Arnold and Sarabia [20]. It is well-known (Belzunce et al. [15]) that

$$
X \leq_{*} Y \Longrightarrow X \leq_{p s} Y .
$$

Section 4 contains further remarks.

\section{Main Results}

Let $X_{1}, \ldots, X_{n}$ be independent and identical copies of a non-negative random variable $X$ with distribution function $F$ and a finite mean $\mu_{X}$. The distribution function of the $i$ th order statistic $X_{i: n}(i=1, \ldots, n)$ is

$$
F_{i: n}(x)=\beta_{i, n-i+1}(F(x)), x \geq 0,
$$

where

$$
\beta_{i, j}(p)=\int_{0}^{p} \frac{(i+j-1) !}{(i-1) !(j-1) !} t^{i-1}(1-t)^{j-1} d t, 0 \leq p \leq 1,
$$

is the Pearson's incomplete beta function (see Pearson [26]) with parameters $(i, j)$.

If $F$ is absolutely continuous with density function $f$, the density function of $X_{i: n}$, $(i=1, \ldots, n)$ is

$$
f_{i: n}(x)=\frac{n !}{(i-1) !(n-i) !}[F(x)]^{i-1}[\bar{F}(x)]^{n-i} f(x), x \geq 0 .
$$

We require the following result due to Barlow and Proschan [27].

Lemma 1. Let $W$ be a measure on the interval $(a, b)$, not necessarily non-negative. Let $h$ be a non-negative function defined on $(a, b)$. If $\int_{x}^{b} d W(t) \geq 0$ for all $x \in(a, b)$ and if $h$ is increasing, then $\int_{a}^{b} h(t) d W(t) \geq 0$.

These represent the conditions required to prove the following result. 
Theorem 1. Let $X$ and $Y$ be non-negative random variables with finite means and continuous distribution functions $F$ and $G$, respectively. Let $X_{1}, \ldots, X_{n}$ be i.i.d. as $X$ and let $Y_{1}, \ldots, Y_{n}$ be i.i.d. as $Y$. Given $1 \leq k \leq n$, if $X_{k: n} \leq{ }_{p s} Y_{k: n}$ then

(a) $X_{j: n} \leq p s Y_{j: n}$, for all $k+1 \leq j \leq n$.

(b) $\quad X_{k: m} \leq p s Y_{k: m}$, for all $k \leq m \leq n-1$.

Proof. We first prove (a). The condition $X_{k: n} \leq_{p s} Y_{k: n}$ is equivalent to

$$
\int_{p}^{1} \frac{F_{k: n}^{-1}(t)}{F_{k: n}^{-1}(p)} d t \leq \int_{p}^{1} \frac{G_{k: n}^{-1}(t)}{G_{k: n}^{-1}(p)} d t, \quad \text { for all } p \in(0,1),
$$

where $F_{k: n}^{-1}(t)$ and $G_{k: n}^{-1}(t)$ are the quantile functions of $X_{k: n}$ and $Y_{k: n}$, respectively. Using

$$
F_{k: n}^{-1}(t)=F^{-1}\left(\beta_{k, n-k+1}^{-1}(t)\right), \quad t \in(0,1),
$$

with $\beta_{i, j}(t)$, the incomplete beta function given by (7) (and similarly for $G_{k: n}^{-1}(t)$ ), we see that (8) holds if and only if

$$
\int_{p}^{1}\left(\frac{G^{-1}\left(\beta_{k, n-k+1}^{-1}(t)\right)}{G^{-1}\left(\beta_{k, n-k+1}^{-1}(p)\right)}-\frac{F^{-1}\left(\beta_{k, n-k+1}^{-1}(t)\right)}{F^{-1}\left(\beta_{k, n-k+1}^{-1}(p)\right)}\right) d t \geq 0, \quad \text { for all } p \in(0,1) .
$$

Making the change of variable $\beta_{k, n-k+1}^{-1}(t)=u$, the latter becomes

$$
\int_{p}^{1}\left(\frac{G^{-1}(u)}{G^{-1}(p)}-\frac{F^{-1}(u)}{F^{-1}(p)}\right) d \beta_{k, n-k+1}(u) \geq 0, \quad p \in(0,1) .
$$

Let us consider $j \geq k+1$ and the non-negative increasing function

$$
h(u)=\frac{(k-1) !(n-k) !}{(j-1) !(n-j) !}\left(\frac{1}{u}-1\right)^{-(j-k)}, u \in[p, 1)
$$

and $h(u)=0$ for $u \in(0, p)$. Since $d \beta_{j, n-j+1}(u)=h(u) d \beta_{k, n-k+1}(u), u \in[p, 1)$, it follows from (10) and Lemma 1 that

$$
\int_{p}^{1}\left(\frac{G^{-1}(u)}{G^{-1}(p)}-\frac{F^{-1}(u)}{F^{-1}(p)}\right) d \beta_{j, n-j+1}(u) \geq 0, \quad p \in(0,1),
$$

which means, using the same argument as before, that $X_{j: n} \leq_{p s} Y_{j: n}$ for all $k+1 \leq j \leq n$.

The proof of (b) follows the same steps as the proof of (a) using $d \beta_{k, m-k+1}(u)=$ $h^{*}(u) d \beta_{k, n-k+1}(u), u \in[p, 1)$, where, given $k \leq m<n$,

$$
h^{*}(u)=\frac{m !(n-k) !}{n !(m-k) !}(1-u)^{-(n-m)}, u \in[p, 1)
$$

and $h^{*}(u)=0$ for $u \in(0, p)$.

As a direct consequence of Theorem 1, we note that

$$
X_{1: n} \leq_{p s} Y_{1: n} \Longrightarrow X_{j: n} \leq_{p s} Y_{j: n} \Longrightarrow X_{j: m} \leq_{p s} Y_{j: m}, 1 \leq j \leq m \leq n .
$$

In particular,

$$
X_{1: n} \leq_{p s} Y_{1: n} \text { for some } n \geq 1 \Longrightarrow X \leq_{p s} Y \text {. }
$$


It is well-known (Belzunce et al. [15]) that

$$
X \leq_{p s} Y \Longleftrightarrow\left(\frac{X-F^{-1}(p)}{F^{-1}(p)}\right)^{+} \leq_{i c x}\left(\frac{Y-G^{-1}(p)}{G^{-1}(p)}\right)^{+}, p \in(0,1),
$$

where $x^{+}=\max \{x, 0\}$.

Next, we state two lemmas that will be used in the proofs of the main results. The first one involves some kind of parallelism with (12) using order statistics. The second one is a technical lemma.

Lemma 2. Let $X$ and $Y$ be non-negative random variables with finite means and continuous distribution functions $F$ and $G$, respectively. Let $X_{1}, \ldots, X_{n}$ be i.i.d. as $X$ and let $Y_{1}, Y_{2}, \ldots, Y_{n}$ be i.i.d. as $Y$. Let $1 \leq k \leq n$, and then $X_{k: n} \leq{ }_{p s} Y_{k: n}$ if and only if

$$
\left(\frac{X-F^{-1}(p)}{F^{-1}(p)}\right)_{k: n}^{+} \leq_{i c x}\left(\frac{Y-G^{-1}(p)}{G^{-1}(p)}\right)_{k: n}^{+} \text {for all } p \in(0,1) .
$$

Proof. It follows from (12) that $X_{k: n} \leq_{p s} Y_{k: n}$ if and only if

$$
\left(\frac{X_{k: n}-F_{k: n}^{-1}(p)}{F_{k: n}^{-1}(p)}\right)^{+} \leq_{i c x}\left(\frac{Y_{k: n}-G_{k: n}^{-1}(p)}{G_{k: n}^{-1}(p)}\right)^{+} \text {for all } p \in(0,1) .
$$

Using (9), this is the same as

$$
\left(\frac{X_{k: n}-F^{-1}(p)}{F^{-1}(p)}\right)^{+} \leq_{i c x}\left(\frac{Y_{k: n}-G^{-1}(p)}{G^{-1}(p)}\right)^{+} \text {for all } p \in(0,1) .
$$
as (13).

Since the function $\left(\frac{x-c}{c}\right)^{+}$(with $c>0$ constant) is increasing, the latter is the same

Lemma 3. Let $X$ and $Y$ be non-negative random variables with finite means and continuous distribution functions $F$ and $G$, respectively. Let $X_{1}, \ldots, X_{n}$ be i.i.d. as $X$ and let $Y_{1}, \ldots, Y_{n}$ be i.i.d. as $Y$. Let $1 \leq k \leq n$, if $X_{k: n} \leq p s Y_{k: n}$, then

$$
\frac{\left(X-F^{-1}(p) \mid X>F^{-1}(p)\right)_{k: n}}{F^{-1}(p)} \leq_{i c x} \frac{\left(Y-G^{-1}(p) \mid Y>G^{-1}(p)\right)_{k: n}}{G^{-1}(p)},
$$

for all $p \in(0,1)$.

Proof. From (9) and Lemma 2, it follows that $X_{k: n} \leq_{p s} Y_{k: n}$ implies

$$
\int_{q}^{1}\left(\frac{G^{-1}\left(\beta_{k, n-k+1}^{-1}(u)\right)}{G^{-1}(p)}-\frac{F^{-1}\left(\beta_{k, n-k+1}^{-1}(u)\right)}{F^{-1}(p)}\right) d u \geq 0,
$$

for all $q \in(0,1)$ and $p \in(0,1)$. The change of variable $\beta_{k, n-k+1}^{-1}(u)=t$ yields

$$
\int_{q}^{1}\left(\frac{G^{-1}(t)}{G^{-1}(p)}-\frac{F^{-1}(t)}{F^{-1}(p)}\right) d \beta_{k, n-k+1}(t) \geq 0,
$$

for all $q \in(0,1)$ and $p \in(0,1)$. Now denote

$$
X_{p}^{*}=\frac{\left(X-F^{-1}(p) \mid X>F^{-1}(p)\right)}{F^{-1}(p)}, \quad p \in(0,1)
$$


and let $F_{X_{p}^{*}}^{-1}$ be its quantile function given by

$$
F_{X_{p}^{*}}^{-1}(t)=\frac{F^{-1}(p+(1-p) t)}{F^{-1}(p)}-1, \quad t \in(0,1) .
$$

The condition

$$
\left(X_{p}^{*}\right)_{k: n} \leq_{i c x}\left(Y_{p}^{*}\right)_{k: n} \text { for all } p \in(0,1)
$$

is equivalent to

$$
\begin{aligned}
& \int_{q}^{1}\left(\frac{F^{-1}\left(p+(1-p) \beta_{k, n-k+1}^{-1}(u)\right)}{F^{-1}(p)}\right) d u \\
& \leq \int_{q}^{1}\left(\frac{G^{-1}\left(p+(1-p) \beta_{k, n-k+1}^{-1}(u)\right)}{G^{-1}(p)}\right) d u,
\end{aligned}
$$

for all $q \in(0,1)$ and $p \in(0,1)$. The change of variable $u=\beta_{k, n-k+1}\left(\frac{t-p}{1-p}\right)$ gives

$$
\int_{q_{p}}^{1}\left(\frac{G^{-1}(t)}{G^{-1}(p)}-\frac{F^{-1}(t)}{F^{-1}(p)}\right) d \beta_{k, n-k+1}\left(\frac{t-p}{1-p}\right) \geq 0,
$$

for all $q \in(0,1)$ and $p \in(0,1)$, where we denoted $q_{p}=p+(1-p) \beta_{k, n-k+1}^{-1}(q)$. To complete the proof note that for each fixed $p$, (16) follows from (14) via Lemma 1 using

$$
d \beta_{k, n-k+1}\left(\frac{t-p}{1-p}\right)=h_{p}(t) d \beta_{k, n-k+1}(t), \quad t \in\left[q_{p}, 1\right),
$$

where $h_{p}$ is the non-negative increasing function

$$
h_{p}(t)= \begin{cases}\frac{1}{(1-p)^{n}}\left(1-\frac{p}{t}\right)^{k-1}, & t \in\left[q_{p}, 1\right) \\ 0, & t \in\left(0, q_{p}\right) .\end{cases}
$$

Now, we are ready to prove the main result of this section, which extends (when restricting to the case of order statistics) Theorem 3.10 of Belzunce et al. [12].

Theorem 2. Let $X$ and $Y$ be non-negative random variables with finite means and absolutely continuous distribution functions $F$ and $G$, respectively. Let $X_{1}, \ldots, X_{n}$ be i.i.d. as $X$ and let $Y_{1}, \ldots, Y_{n}$ be i.i.d. as $Y$. Given $1 \leq i<j \leq n$,

(a) $X_{j-i: n-i} \leq_{p s} Y_{j-i: n-i}$ implies $R_{i, j, n}^{X} \leq_{i c x} R_{i, j, n}^{Y}$;

(b) $\quad X_{1: n-i} \leq_{p s} Y_{1: n-i}$ implies $R_{i, j, n}^{X} \leq_{i c x} R_{i, j, n^{\prime}}^{Y}$

where $R_{i, j, n}^{X}$ is defined by (2).

Proof. Let $1 \leq i<j \leq n$. Using Theorem 2.4.1 in Arnold et al. [28], we note that the survival function of the random variable $R_{i, j, n}^{X}$ is given by

$$
\begin{aligned}
\bar{F}_{R_{i, j, n}}(x) & =\int_{0}^{\infty} P\left(X_{j: n}>(x+1) u \mid X_{i: n}=u\right) f_{i: n}(u) d u \\
& =\int_{0}^{\infty} P\left((X \mid X>u)_{j-i: n-i}>u(x+1)\right) d \beta_{i, n-i+1}(F(u)) .
\end{aligned}
$$


The change of variable $F(u)=p$ yields

$$
\bar{F}_{R_{i, j, n}}(x)=\int_{0}^{1} \bar{F}_{j-i: n-i}^{X_{p}^{*}}(x) d \beta_{i, n-i+1}(p),
$$

where $X_{p}^{*}$ is given by (15). Consequently, $R_{i, j, n}^{X} \leq_{i c x} R_{i, j, n}^{Y}$ holds if and only if

$$
\begin{aligned}
& \int_{0}^{1}\left(\int_{\mathcal{S}}^{\infty} \bar{F}_{j-i: n-i}^{X_{p}^{*}}(x) d x\right) d \beta_{i, n-i+1}(p) \\
\leq & \int_{0}^{1}\left(\int_{\mathcal{S}}^{\infty} \bar{G}_{j-i: n-i}^{Y_{p}^{*}}(x) d x\right) d \beta_{i, n-i+1}(p), \quad \text { for all } s .
\end{aligned}
$$

Now, to prove part (a), assume $X_{j-i: n-i} \leq_{p s} Y_{j-i: n-i}$. It follows from Lemma 3 that

$$
\left(X_{p}^{*}\right)_{j-i: n-i} \leq_{i c x}\left(Y_{p}^{*}\right)_{j-i: n-i} \text { for all } p \in(0,1)
$$

that is,

$$
\int_{s}^{\infty} \bar{F}_{j-i: n-i}^{X_{p}^{*}}(x) d x \leq \int_{s}^{\infty} \bar{G}_{j-i: n-i}^{Y_{p}^{*}}(x) d x, \text { for all } s
$$

and the result holds via (18). To prove (b), assume $X_{1: n-i} \leq_{p s} Y_{1: n-i}$. It follows from Theorem 1 (a) that $X_{j-i: n-i} \leq_{p s} Y_{j-i: n-i}$, and the result follows by applying part (a).

Many authors have investigated conditions involving various stochastic orders, under which two ordered random variables belong to the same equivalence class. Given two random variables $X$ and $Y$ and a certain stochastic order $\leq_{\text {ord }}$, these results are usually of the form

$$
X \leq_{\text {ord }} Y \text { and } T(X)=T(Y) \text {, then } X \equiv_{\text {ord }} Y,
$$

where $T$ is a characteristic, such as the mean, the variance, and others (see, for example, Section 3 in Sordo [29] and references therein). In this vein, we provide a sufficient condition for stochastic equality (up to a scale parameter) of two $\leq_{p s}$-ordered random variables (the expected proportional shortfall order is scale-free).

Theorem 3. Let $X$ and $Y$ be non-negative random variables with finite means and absolutely continuous distribution functions $F$ and $G$, respectively. Let $X_{1}, \ldots, X_{n}$ be i.i.d. as $X$ and let $Y_{1}, \ldots, Y_{n}$ be i.i.d. as $Y$. Given $1 \leq i<n$, if $X_{1: n-i} \leq_{p s} Y_{1: n-i}$ and

$$
E\left[\frac{X_{i+1: n}-X_{i: n}}{X_{i: n}}\right]=E\left[\frac{Y_{i+1: n}-Y_{i: n}}{Y_{i: n}}\right]
$$

then $X \equiv_{s t} c \cdot Y$, for some $c>0$.

Proof. From (17), we can write

$$
\begin{aligned}
\bar{F}_{R_{i, i+1, n}}(x) & =\int_{0}^{1} \bar{F}_{1: n-i}^{X_{p}^{*}}(x) d \beta_{i, n-i+1}(p) \\
& =\int_{0}^{1}\left[\frac{\bar{F}\left((1+x) F^{-1}(p)\right)}{1-p}\right]^{n-i} d \beta_{i, n-i+1}(p) \\
& =\left(\begin{array}{c}
n \\
i
\end{array}\right) \int_{0}^{1}\left[\bar{F}\left((1+x) F^{-1}(p)\right)\right]^{n-i} d \beta_{i, 1}(p),
\end{aligned}
$$


where we used

$$
\begin{aligned}
d \beta_{i, n-i+1}(p) & =\frac{n !}{(i-1) !(n-i) !} p^{i-1}(1-p)^{n-i} d p \\
& =\left(\begin{array}{c}
n \\
i
\end{array}\right)(1-p)^{n-i} d \beta_{i, 1}(p) .
\end{aligned}
$$

Rewriting (19), we see that

$$
\bar{F}_{R_{i, i+1, n}}(x)=\left(\begin{array}{c}
n \\
i
\end{array}\right) \int_{0}^{1} \bar{F}\left(\left(x-F^{-1}(p)\right) / F^{-1}(p)\right)_{1: n-i}^{+}(x) d \beta_{i, 1}(p) .
$$

Making the change of variable $p=\beta_{1, n-i}^{-1}(t)$ and using the same argument as in the proof of Lemma 2, we have

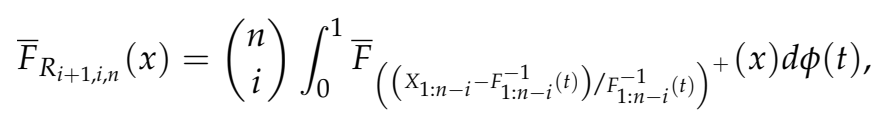

where

$$
\phi(t)=\beta_{i, 1}\left(\beta_{1, n-i}^{-1}(t)\right)=\left(1-(1-t)^{1 /(n-i)}\right)^{i} .
$$

Now, given $1 \leq i<n$, we have

$$
\begin{aligned}
E\left[\frac{X_{i+1: n}-X_{i: n}}{X_{i: n}}\right] & =\int_{0}^{\infty} \bar{F}_{R_{i+1, i, n}}(x) d x \\
& =\left(\begin{array}{c}
n \\
i
\end{array}\right) \int_{0}^{\infty}\left(\int_{0}^{1} \bar{F}\left(\left(x_{1: n-i}-F_{1: n-i}^{-1}(t)\right) / F_{1: n-i}^{-1}(t)\right)\right. \\
& =\left(\begin{array}{c}
n \\
i
\end{array}\right) \int_{0}^{1}\left(\int_{0}^{\infty} \bar{F}\left(\left(x_{1: n-i}-F_{1: n-i}^{-1}(t)\right) / F_{1: n-i}^{-1}(t)\right)\right. \\
& +(x) d x) d \phi(t) d x \\
& =\left(\begin{array}{c}
n \\
i
\end{array}\right) \int_{0}^{1} A_{1: n-i}^{X}(t) d \phi(t),
\end{aligned}
$$

where

$$
A_{1: n-i}^{X}(t)=\frac{\int_{F_{1: n-i}^{-1}(t)}^{\infty} \bar{F}_{1: n-i}(x) d x}{F_{1: n-i}^{-1}(t)} .
$$

From the assumptions, we note that

$$
A_{1: n-i}^{X}(t) \leq A_{1: n-i}^{Y}(t), \text { for all } t \in(0,1)
$$

and

$$
\int_{0}^{1}\left(A_{1: n-i}^{Y}(t)-A_{1: n-i}^{X}(t)\right) d \phi(t)=0 .
$$

Combining (20) and (21) and taking into account the strictly monotony of $\phi$, it follows that $A_{1: n-i}^{X}(t)=A_{1: n-i}^{Y}(t)$ for all $t \in(0,1)$. Since the expected proportional shortfall order is scale-invariant (see Belzunce et al. [15]), this holds if and only if

$$
\bar{F}_{1: n-i}(t)=\bar{G}_{1: n-i}(c \cdot t), \text { for some } c>0, \text { for all } t,
$$

which is equivalent to

$$
(\bar{F}(t))^{n-i}=(\bar{G}(c \cdot t))^{n-i}, \text { for some } c>0, \text { for all } t .
$$

This means that $X$ and $Y$ have the same distribution up to a scale parameter, which ends the proof. 
By taking $i=n-1$ in Theorem 3, we obtain the corollary presented below.

Corollary 1. Let $X$ and $Y$ be two non-negative random variables with finite means and absolutely continuous distribution functions $F$ and $G$, respectively. Let $X_{1}, \ldots, X_{n}$ be i.i.d. as $X$ and let $Y_{1}, \ldots, Y_{n}$ be i.i.d. as $Y$. If $X \leq_{p s} Y$ and

$$
E\left[\frac{X_{n: n}-X_{n-1: n}}{X_{n-1: n}}\right]=E\left[\frac{Y_{n: n}-Y_{n-1: n}}{Y_{n-1: n}}\right]
$$

then $X \equiv_{s t} c \cdot Y$, for some $c>0$.

Remark 1. Under the assumption $X \leq_{p s} Y$, Corollary 1 can be used to build a test for the null hypothesis

$$
H_{0}: X={ }_{s t} c Y, \text { for some } c>0,
$$

against the alternative

$$
H_{1}: X \leq_{p s} Y \text { and } X \neq_{s t} c Y \text { for all } c>0 .
$$

Clearly, the functional $\Delta=E\left[\frac{Y_{n: n}-Y_{n-1: n}}{Y_{n-1: n}}\right]-E\left[\frac{X_{n: n}-X_{n-1: n}}{X_{n-1: n}}\right]$ is a measure of departure from $H_{0}$ in favor of $H_{1}$. Corollary 1 suggests to reject the null hypothesis if $\hat{\Delta}(n, m)>k$, where $\hat{\Delta}(n, m)$ is an estimator of $\Delta$ based on two random samples $\left(x_{1}, \ldots, x_{n}\right)$ and $\left(y_{1}, \ldots, y_{m}\right)$ drawn from $X$ and $Y$, respectively, and $k$ depends on the null distribution of $\hat{\Delta}(n, m)$.

\section{Application to the Comparison of Parametric Families of Income Distributions in Terms of Relative Deprivation}

Let $X$ be an income random variable with distribution function $F$ and let $X_{1: n}, \ldots, X_{n: n}$ be the corresponding order statistics. As explained in Section 1, the expectation of

$$
R D P_{i, n}^{X}=\frac{1}{n} \sum_{j=i+1}^{n}\left(\frac{X_{j: n}-X_{i: n}}{X_{i: n}}\right)=\frac{1}{n} \sum_{j=i+1}^{n} R_{i, j, n}^{X}
$$

is a measure of relative deprivation of $X$. According to Chakravarty and Moyes [24], we say that there is no more relative deprivation in situation $X$ than in $Y$ (denoted by $X \geq_{R D P} Y$ ) if

$$
E\left[R D P_{i, n}^{X}\right] \leq E\left[R D P_{i, n}^{Y}\right], \text { for } i=1,2, \ldots, n-1 .
$$

We obtain the result presented below.

Corollary 2. Let $X$ and $Y$ be two random variables with finite means and absolutely continuous distribution functions $F$ and $G$, respectively. Let $X_{1}, \ldots, X_{n}$ be i.i.d. as $X$ and let $Y_{1}, \ldots, Y_{n}$ be i.i.d. as $Y$. If $X_{1: n-1} \leq_{p s} Y_{1: n-1}$ for some $n \geq 2$, then $X \geq_{R D P} Y$.

Proof. If $X_{1: n-1} \leq_{p s} Y_{1: n-1}$, it follows from Theorem 1 (b) that $X_{1: n-i} \leq_{p s} Y_{1: n-i}$, for all $1 \leq i \leq n-1$ and from Theorem 2 (b) that $R_{i, j, n}^{X} \leq_{i c x} R_{i, j, n}^{Y}$ for $i=1, \ldots, n-1$ and $j=i+1, \ldots, n$. The result follows using that $R_{i, j, n}^{X} \leq_{i c x} R_{i, j, n}^{Y}$ implies $E\left[R_{i, j, n}^{X}\right] \leq E\left[R_{i, j, n}^{Y}\right]$.

Since, in view of Corollary 2, the condition $X_{1: n} \leq_{p s} Y_{1: n}$ is useful in comparing income distributions in terms of relative deprivation, we characterize this condition within several parametric families. We focus on the generalized gamma (GG) and generalized beta of the second kind (GB2), which include, as particular cases, some of the most popular distributions used in the analysis of income. They are defined in terms of their densities as follows (see Chapter 10 of the book by Arnold and Sarabia [20] for a review and Sarabia et al. [30] for some recent applications).

Definition 3. Given a random variable $X$, we say that 
(i) X follows a generalized gamma distribution with parameters $a, b, p>0$ (denoted by $X \sim$ $G G(a, b, p))$ if its density function is given by

$$
f(x)=\frac{a x^{a p-1} e^{-(x / b)^{a}}}{b^{a p} \Gamma(p)}, \quad \text { for all } x>0,
$$

where $\Gamma(p)=\int_{0}^{\infty} z^{p-1} e^{-z} d z$ is the gamma function.

(ii) $X$ follows a type II generalized beta distribution with parameters $a, b, p, q>0$ (denoted by $X \sim G B 2(a, b, p, q))$ if its density function is given by

$$
f(x)=\frac{a x^{a p-1}}{b^{a p} \beta(p, q)\left[1+(x / b)^{a}\right]^{p+q}}, \quad \text { for all } x>0,
$$

where $\beta(p, q)=\Gamma(p) \Gamma(q) / \Gamma(p+q)$ is the beta function.

First, we characterize the condition $X_{1: n} \leq_{p s} Y_{1: n}$ within the generalized gamma family. Particular members of this family are gamma $(a=1)$ and Weibull $(p=1)$.

Corollary 3. Let $X \sim G G\left(a_{1}, b_{1}, p_{1}\right)$ and $Y \sim G G\left(a_{2}, b_{2}, p_{2}\right)$ be two income distributions. Then, $X_{1: n} \leq_{p s} Y_{1: n}$ if and only if $a_{1} \geq a_{2}$ and $a_{1} p_{1} \geq a_{2} p_{2}$.

Proof. First, note that the role of the parameters $b_{1}$ and $b_{2}$ is irrelevant since they are scale parameters and the expected proportional shortfall order is scale-invariant. Now, assume $X_{1: n} \leq_{p s} Y_{1: n}$. It follows from (11) that $X \leq_{p s} Y$, which holds (Theorem 3.1 in Belzunce et al. [16]) if and only if $a_{1} \geq a_{2}$ and $a_{1} p_{1} \geq a_{2} p_{2}$. Conversely, the assumptions $a_{1} \geq a_{2}$ and $a_{1} p_{1} \geq a_{2} p_{2}$ imply $X \leq_{*} Y$ (Theorem 3.1 in Belzunce et al. [16]). This is equivalent, using $F_{1: n}^{-1}(p)=F^{-1}\left(\beta_{1, n}^{-1}(p)\right)$, to $X_{1: n} \leq_{*} Y_{1: n}$. From (6), it follows that $X_{1: n} \leq_{p s} Y_{1: n}$.

The proofs of the following results follow the same lines as the proof of Corollary 3 , using corollaries 3.7 to 3.11 of Belzunce et al. [16]. The distributions under consideration are particular members of the $G B 2$ family, namely, type II beta distributions $(G B 2(1, p, q))$, Singh-Maddala distribution $(G B 2(a, 1, q))$, Dagum distribution $(G B 2(a, p, 1))$, Lomax distribution $(L(q)=G B 2(1,1, q))$, and Fisk distribution $(G B 2(a, 1,1))$.

Corollary 4. Let $X \sim G B 2\left(1, p_{1}, q_{1}\right)$ and $Y \sim G B 2\left(1, p_{2}, q_{2}\right)$ be two income distributions with $q_{i}>1$ for $i=1$, 2. Then, $X_{1: n} \leq{ }_{p s} Y_{1: n}$ if and only if $p_{1} \geq p_{2}$ and $q_{1} \geq q_{2}$.

Corollary 5. Let $X \sim G B 2\left(a_{1}, 1, q_{1}\right)$ and $Y \sim G B 2\left(a_{2}, 1, q_{2}\right)$ be two income distributions with $a_{i} q_{i}>1$ for $i=1,2$. Then, $X_{1: n} \leq p s Y_{1: n}$ if and only if $a_{1} \geq a_{2}$ and $a_{1} q_{1} \geq a_{2} q_{2}$.

Corollary 6. Let $X \sim G B 2\left(a_{1}, p_{1}, 1\right)$ and $Y \sim G B 2\left(a_{2}, p_{2}, 1\right)$ be two income distributions with $a_{i}>1$ for $i=1,2$. Then, $X_{1: n} \leq p s Y_{1: n}$ if and only if $a_{1} \geq a_{2}$ and $a_{1} p_{1} \geq a_{2} p_{2}$.

Corollary 7. Let $X \sim G B 2\left(1,1, q_{1}\right)$ and $Y \sim G B 2\left(1,1, q_{2}\right)$ be two income distributions with $q_{i}>1$ for $i=1,2$. Then, $X_{1: n} \leq_{p s} Y_{1: n}$ if and only if $q_{1} \geq q_{2}$.

Corollary 8. Let $X \sim G B 2\left(a_{1}, 1,1\right)$ and $Y \sim G B 2\left(a_{2}, 1,1\right)$ be two income distributions with $a_{i}>1$ for $i=1,2$. Then $X_{1: n} \leq_{p s} Y_{1: n}$ if and only if $a_{1} \geq a_{2}$.

\section{Further Remarks}

The expected proportional shortfall order is related to the excess wealth order, which is defined below. 
Definition 4. Let $X$ and $Y$ be two random variables with respective distribution functions $F$ and $G$; we say that $X$ is less than $Y$ in the excess wealth order (denoted by $X \leq_{e w} Y$ ) if and only if

$$
\int_{F^{-1}(p)}^{\infty} \bar{F}(t) d t \leq \int_{G^{-1}(p)}^{\infty} \bar{G}(t) d t, \text { for all } p \in(0,1) .
$$

For some recent references on this order, see Castaño-Martínez et al. [31], Arriaza and Sordo [32], Wu et al. [33], Toomaj and Di Crescenzo [34,35], and Ortega-Jiménez et al. [36]. The following result is similar to Theorem 2 but in terms of the excess wealth order, and it extends (when restricting to the case of order statistics) Theorem 3.1 of Belzunce et al. [10]. The proof follows the same lines as the proof of Theorem 2; therefore, it is omitted.

Theorem 4. Let $X$ and $Y$ be two random variables with finite means and absolutely continuous distribution functions $F$ and $G$, respectively. Let $X_{1}, \ldots, X_{n}$ be i.i.d. as $X$ and let $Y_{1}, \ldots, Y_{n}$ be i.i.d. as $Y$. Given $1 \leq i<j \leq n$,

(a) $X_{j-i: n-i} \leq_{e w} Y_{j-i: n-i}$ implies $S_{i, j, n}^{X} \leq_{i c x} S_{i, j, n}^{Y}$;

(b) $X_{1: n-i} \leq_{e w} Y_{1: n-i}$ implies $S_{i, j, n}^{X} \leq_{i c x} S_{i, j, n^{\prime}}^{Y}$ where $S_{i, j, n}^{X}$ is defined by (1).

Author Contributions: Writing—review and editing, A.C.-M., G.P. and M.A.S. All authors have read and agreed to the published version of the manuscript.

Funding: This research was partially funded by Ministerio de Economía y Competitividad (Spain) under grant MTM2013-46962-C2-2-P and by the 2014-2020 ERDF Operational Programme and the Department of Economy, Knowledge, Business and University of the Regional Government of Andalusia under grant FEDER-UCA18-107519.

Institutional Review Board Statement: Not applicable.

Informed Consent Statement: Not applicable.

Data Availability Statement: Not applicable.

Conflicts of Interest: The authors declare no conflicts of interest.

\section{References}

1. Kochar, S.C. Some partial ordering results on record values. Commun. Stat. Theory Methods 1990, 19, 299-306. [CrossRef]

2. Kochar, S.C.; Rojo, J. Some new results on stochastic comparisons of spacings from heterogeneous exponential distributions. J. Multivar. Anal. 1996, 59, 272-281. [CrossRef]

3. Hu, T.; Wei, Y. Stochastic comparisons of spacings from restricted families of distributions. Stat. Probab. Lett. 2001, 53, 91-99. [CrossRef]

4. Kochar, S.C.; Li, X.; Xu, M. Excess wealth order and sample spacings. Statist. Methodol. 2007, 4, 385-392. [CrossRef]

5. Kochar, S.C.; Xu, M. Stochastic comparisons of parallel systems when components have proportional hazard rates. Probab. Eng. Inf. Sci. 2007, 21, 597-609. [CrossRef]

6. Genest, C.; Kochar, S.C.; Xu, M. On the range of heterogeneous samples. J. Multivar. Anal. 2009, 100, 1587-1592. [CrossRef]

7. Mao, T.; Hu, T. Equivalent characterizations on orderings of order statistics and sample ranges. Probab. Eng. Inf. Sci. 2010, 24, 245-262. [CrossRef]

8. Zhao, P.; Zhang, Y. On sample ranges in multiple-outlier models. J. Multivar. Anal. 2012, 111, 335-349. [CrossRef]

9. Torrado, N.; Lillo, R. Likelihood ratio comparisons among spacings related to both one or two samples. Statistics 2015, 19, 831-841. [CrossRef]

10. Belzunce, F.; Martínez-Riquelme, C.; Ruiz, J.M.; Sordo, M.A. On sufficient conditions for the comparison in the excess wealth order and spacings. J. Appl. Probab. 2016, 53, 33-46. [CrossRef]

11. Ding, W.; Zhang, Y.; Zhao, P. Ordering properties of spacings from heterogeneous geometric samples. Probab. Eng. Inf. Sci. 2018, 32, 306-322. [CrossRef]

12. Belzunce, F.; Martínez-Riquelme, C.; Ruiz, J.M.; Sordo, M.A. On the comparison of relative spacings with applications. Methodol. Comput. Appl. Probab. 2017, 19, 357-376. [CrossRef]

13. Shaked, M.; Shanthikumar, J.G. Stochastic Orders; Springer Series in Statistics; Springer: New York, NY, USA, 2007.

14. Belzunce, F.; Martínez-Riquelme, C.; Mulero, J. An Introduction to Stochastic Orders; Academic Press: Cambridge, MA, USA, 2015. 
15. Belzunce, F.; Pinar, J.F.; Ruiz, J.M.; Sordo, M.A. Comparison of risks based on the expected proportional shortfall. Insur. Math. Econ. 2012, 51, 292-302. [CrossRef]

16. Belzunce, F.; Pinar, J.F.; Ruiz, J.M.; Sordo, M.A. Comparison of concentration for several families of income distributions. Stat. Probab. Lett. 2013, 83, 1036-1045. [CrossRef]

17. Di Crescenzo, A.; Martinucci, B.; Mulero, J. A quantile-based probabilistic mean value theorem. Probab. Eng. Inf. Sci. 2016, 30, 261-280. [CrossRef]

18. Belzunce, F.; Martínez-Riquelme, C. On sufficient conditions for the comparison of some quantile based measures. Commun. Stat. Theory Methods 2017, 46, 6512-6527. [CrossRef]

19. Sordo, M.A.; Berihuete, A.; Ramos, C.D.; Ramos, H.M. On a property of Lorenz curves with monotone elasticity and its application to the study of inequality by using tax data. SORT Stat. Oper. Res. Trans. 2017, 41, 55-72.

20. Arnold, B.C.; Sarabia, J.M. Majorization and the Lorenz Order with Applications in Applied Mathematics and Economics; Springer: Cham, Switzerland, 2018.

21. Arriaza, A.; Belzunce, F.; Martínez-Riquelme, C. Sufficient conditions for some transform orders based on the quantile density ratio. Methodol. Comput. Appl. Probab. 2019, 23, 29-52. [CrossRef]

22. Runciman, W.G. Relative Deprivation and Social Justice; Routledge and Kegan Paul: London, UK, 1966.

23. Paul, S. An index of relative deprivation. Econ. Lett. 1991, 36, 337-341. [CrossRef]

24. Chakravarty, S.R.; Moyes, P. Individual welfare, social deprivationand income taxation. Econ. Theory 2003, 21, 843-869. [CrossRef]

25. Chakravarty, S.R.; D'Ambrosio, C.; Muliere, P. Target Shortfall Orderings and Indices; DIW Discussion Papers 340; German Institute for Economic Research: Berlin, Germany, 2003.

26. Pearson, K. Tables of the Incomplete Beta Function; Cambridge University Press: Cambridge, UK, 1934.

27. Barlow, R.E.; Proschan, F. Mathematical Theory of Reliability; John Wiley \& Sons: New York, NY, USA, 1975.

28. Arnold, B.C.; Balakrishnan, N.; Nagaraja H.N. A First Course in Order Statistics; Wiley: New York, NY, USA, 2008.

29. Sordo, M.A. Characterizations of classes of risk measures by dispersive orders. Insur. Math. Econ. 2008, 42, 1028-1034. [CrossRef]

30. Sarabia, J.M.; Jordá, V.;Prieto, F.; Guillén, M. Multivariate Classes of GB2 Distributions with Applications. Mathematics 2021, 9 , 72. [CrossRef]

31. Castaño-Martínez, A.; Pigueiras, G.; Sordo, M.A. On a family of risk measures based on largest claims. Insur. Math. Econ. 2019, 86, 92-97. [CrossRef]

32. Arriaza, A.; Sordo, M.A. Preservation of some stochastic orders by distortion functions with application to coherent systems with exchangeable components. Appl. Stoch. Models Bus. Ind. 2020. [CrossRef]

33. Wu, J.; Wang, M.; Li, X. Convex transform order of the maximum of independent Weibull random variables. Statist. Prob. Lett. 2020, 156, 108597. [CrossRef]

34. Toomaj, A.; Di Crescenzo, A. Connections between weighted generalized cumulative residual entropy and variance. Mathematics 2020, 8, 1072. [CrossRef]

35. Toomaj, A.; Di Crescenzo, A. Generalized entropies, variance and applications. Entropy 2020, 22, 709. [CrossRef]

36. Ortega-Jiménez, P; Sordo, M.A.; Suárez-Llorens, A. Stochastic orders and multivariate measures of risk contagion. Insur. Math. Econ. 2021, 96, 199-207. [CrossRef] 